\title{
Insights into Water Interaction at the Interface of Nitrogen- Functionalized Hydrothermal Carbons
}

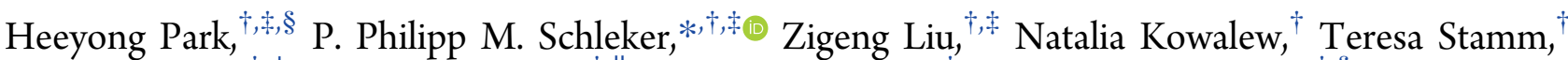 \\ Robert Schlögl, ${ }^{\dagger}, \perp$ Rüdiger-A. Eichel, ${ }^{\ddagger}, \|$ Saskia Heumann, ${ }^{*}+\oplus$ and Josef Granwehr ${ }^{\ddagger}, \S$
}

\footnotetext{
${ }^{\dagger}$ Department of Heterogeneous Reactions, Max Planck Institute for Chemical Energy Conversion, 45470 Mülheim an der Ruhr, Germany

${ }^{\ddagger}$ Forschungszentrum Jülich, IEK-9, 52425 Jülich, Germany

${ }^{\S}$ Institute of Technical and Macromolecular Chemistry and "Institute of Physical Chemistry, RWTH Aachen University, 52074 Aachen, Germany

${ }^{\perp}$ Fritz Haber Institute of the Max Planck Society, 14195 Berlin, Germany
}

\section{Supporting Information}

\begin{abstract}
Hydrothermal carbon (HTC) derived from biomass is a class of cost-efficient, eco-friendly functional carbon materials with various potential applications. In this work, solidstate nuclear magnetic resonance (NMR), longitudinal $\left(T_{1}\right)$ relaxation time, and diffusion NMR were employed to investigate the structure and water dynamics for HTC and nitrogenfunctionalized hydrothermal carbon (N-HTC) samples ((N)HTC). Results showed that the presence of $\mathrm{N}$-functional groups influences the water interaction with (N)-HTC more strongly than surface area, pore size distribution, or oxygenated functional groups. Furthermore, the degree of water interaction can be tuned by adjusting the synthesis temperature and the precursor
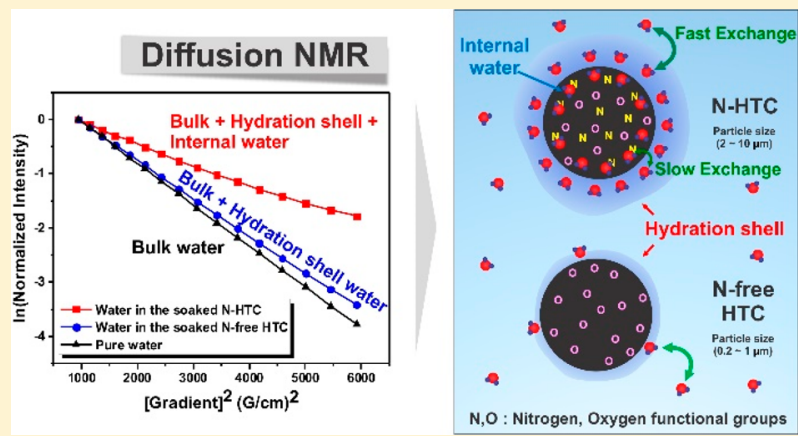
ratio. Water motion was more strongly inhibited in N-HTC than in N-free HTC, thereby suggesting the existence of a differently structured hydration shell around N-HTC particles. In addition, the diffusion data of water in the N-HTC material show two components that do not exchange on the time scale of the experiment (tens of milliseconds), indicating a significant fraction of slow mobile water that exists inside the structure of N-HTC. ${ }^{1} \mathrm{H}-{ }^{2} \mathrm{H}$ isotope exchange and cross-polarization NMR results show this internal water only in a near-surface layer of the N-HTC particles. Based on these findings, a model for water interaction with (N)-HTC particles is proposed.
\end{abstract}

\section{INTRODUCTION}

Carbon materials feature various unique properties, such as high electrical and thermal conductivity, a large variability in crystallinity and morphology, a reasonable corrosion resistance, and low thermal expansion. ${ }^{1,2}$ Their properties make them suitable for a wide range of applications, including catalysts, adsorbers, electrodes, and so on. ${ }^{3-6}$ Therefore, synthesizing theses functionalized active carbon materials from cheap natural precursors through environmentally friendly and lowenergy processes is a very active research topic in materials chemistry today. ${ }^{7}$ One of these carbonaceous materials, the hydrothermal carbon (HTC), is fabricated by a thermochemical pretreatment process using biomass, such as carbohydrates, agricultural residues, or wood chips, in an aqueous medium at mild temperatures, usually between about 130 and $250{ }^{\circ} \mathrm{C}$. This hydrothermal technique is a promising synthetic method allowing for a good control of morphology, chemical composition, and structure. ${ }^{8}$

The addition of functionalized heteroatoms like nitrogen is a popular method in hydrothermal processes to tune both the structural and the physicochemical properties of carbon materials for various desired end applications. ${ }^{9-11}$ Using hydrothermal carbonization for the production of heteroatom-substituted carbons allows chemical reactions to occur between the decomposition products of carbohydrates (such as hydroxymethylfurfural, HMF) and nitrogen precursors through Maillard type chemistry. ${ }^{12}$ Therefore, nitrogen can be covalently bonded to the final carbon structure introducing a large amount of various functional groups. ${ }^{13}$ This is an important advantage for the following pyrolysis step, which is used to improve the electrical conductivity for electrochemical applications. A large part of the nitrogen can be retained even after the high-temperature treatment, while in the classical pyrolytic process of nitrogen-containing precursors most of the nitrogen is lost as volatile species. The formed nitrogencontaining functional groups on carbon materials, such as

Received: June 4, 2019

Revised: August 21, 2019

Published: September 20, 2019 
pyrrole, nitrile, amine, pyridine, imine, amide, lactam, and so on, ${ }^{14}$ can determine the acidic or basic properties and thus the surface reactivity of carbon materials.

Nitrogen-functionalized HTC (N-HTC) is beginning to play an important role in energy conversion and storage technologies such as water splitting. ${ }^{12,15}$ In electrochemistry, there are solid/liquid/gas interfaces that need to be understood to be able to tune the surface properties with a knowledge-based approach. Therefore, the fundamental understanding regarding the chemical structure of materials and the water interaction with its surface are substantial when applying either N-HTC or HTC ( $\mathrm{N})$-HTC) based materials as an electrode for water splitting. Even though the conductivity of unpyrolyzed (N)-HTC samples used in this paper is not high enough to be used directly as an electrode, the structure after the initial synthesis step affects that of the final product and also represents its lower boundary. Tuning of the structure is therefore most effective by optimizing the synthesis parameters rather than by adjusting the pyrolysis step. It is important to understand relationship between structure and water interaction at this intermediate stage.

Various methods have been used to investigate the structure of carbonaceous materials and their interaction with surrounding fluids, including chemical decomposition, thermal decomposition, or spectroscopic techniques such as infrared (IR), Raman, fluorescence, or nuclear magnetic resonance (NMR). ${ }^{12,16-20}$ Solid-state NMR and molecular dynamics NMR represent a nondestructive, noninvasive way to identify correlations between the chemical structure of materials and their properties. ${ }^{21-26}$ Based on solid-state NMR spectroscopy, structural comparisons of different (N)-HTCs synthesized from glucose and urotropine were reported. ${ }^{12}$ The whole ${ }^{13} \mathrm{C}$ NMR spectral region could be classified chiefly into three parts: (i) $\mathrm{sp}^{3}$-aliphatic carbon atoms (0-95 ppm), (ii) $\mathrm{sp}^{2}$ carbon atoms including aromatic and carboxylic groups (95$188 \mathrm{ppm}$ ), and (iii) carbonyl groups, especially ketones and aldehydes (188-230 ppm). The furan-based moieties were the major structural motifs in which aliphatic compounds acted as linkers within the polymeric carbonaceous network. However, when increasing the molar ratio of urotropine, the furanic structures disappeared and were replaced by $\mathrm{N}$-containing moieties. $^{12}$

When water molecules are located in pores and interact with functional groups at the surface of materials, their motion is restricted. This phenomenon can be detected by NMR relaxation and diffusion measurements. In previous studies, low-field NMR relaxometry with fast field cycling (FFC NMR) was conducted to reveal the nature of the interactions between water and chars depending on the pore size distribution of the chars. ${ }^{27}$ However, these studies were performed only for chemically uniform chars and did not provide information about the dynamics of water in chars with different chemical compositions. In a recent paper, it was reported that the chemical composition of hydrochar and pyrochar is not important for the dynamics of water, but its contribution to the strength of the water-solid phase interaction is not yet clear. ${ }^{28}$

In this paper, the water interaction characteristics of $(\mathrm{N})$ HTC materials before the pyrolysis step are analyzed. The water interaction can be fine-tuned by varying the nitrogen substitution on HTC material, which is achieved by adjusting the synthesis temperature and the ratio of precursors. The importance of $\mathrm{N}$-functional groups for water mobility is compared with other influence factors such as surface area, pore size distribution, and the oxygenated functional groups through NMR relaxation and diffusion measurements. Finally, a model is proposed to explain the observed differences in water mobility depending on the presence of nitrogencontaining functional groups in the carbonaceous material.

\section{EXPERIMENTAL SECTION}

Material Synthesis. HTC and N-HTC samples were synthesized by using pure glucose and a mixture of glucose and urotropine, respectively. ${ }^{12}$ All chemicals were of analytical grade. D- $(+)$-Glucose $(\geq 99.5 \%$, Glu) was purchased from Sigma-Aldrich (Steinheim am Albuch, Germany). Hexamethylenetetramine ( $\geq 99 \%$, Urotropine, Uro) was supplied by Carl Roth Chemicals GmbH (Karlsruhe, Germany). Glucose and urotropine were uniformly dissolved in distilled water under stirring. The reaction mixtures were divided into portions of $30 \mathrm{~mL}$ per reactor, Teflon-lined stainless-steel autoclaves (50 $\mathrm{mL}$ volume, Berghof $\mathrm{GmbH}$, Eningen, Germany). The closed reactors were then placed into the associated programmable heating block, preheated to 180 , 220 , or $250^{\circ} \mathrm{C}$ for $6 \mathrm{~h}$. After cooling to room temperature, the solid products were collected by filtration, washed several times with $10 \mathrm{~L}$ of distilled water, and then dried in an oven at $80{ }^{\circ} \mathrm{C}$ for $8 \mathrm{~h}$. In an agate mortar, the (N)-HTCs were finally ground into homogenized powder. All samples and their preparation conditions are summarized in Table S1. The name of each sample include the ratio of Glu/Uro as well as the synthesis temperature.

For the local structure characterization of (N)-HTCs as a function of the synthesis conditions by solid state $\mathrm{NMR},{ }^{13} \mathrm{C}$ labeled glucose (Merck, $\geq 99 \%$ ) and ${ }^{13} \mathrm{C} /{ }^{15} \mathrm{~N}$-labeled urotropine (Merck, $\geq 99 \%$ ) were used to synthesize ${ }^{13} \mathrm{C} /{ }^{15} \mathrm{~N}$ enriched (N)-HTCs with the same procedure as for the nonenriched (N)-HTCs.

Physical Characterizations. Elemental analysis of $\mathrm{C}, \mathrm{H}$, and $\mathrm{N}$ was performed using an Elementar Vario EL CHNOS analyzer. Nitrogen sorption experiments were measured at 77.3 K on a QuadraSorb Station 3 (Quantachrome Instruments). The samples were outgassed for $12 \mathrm{~h}$ at $95{ }^{\circ} \mathrm{C}$ prior to nitrogen sorption analysis. Data were evaluated according to BET theory. ${ }^{29}$ Scanning electron microscopy (SEM) images were acquired on a FESEM SU8020 (HITACHI, Japan) with secondary electron detectors.

NMR Experiments. ${ }^{13} \mathrm{C}$ direct polarization (DP) magic angle spinning (MAS) NMR experiments ${ }^{30}$ were performed on a Bruker (Karlsruhe, Germany) Avance III HD $800 \mathrm{MHz}$ spectrometer using a $3.2 \mathrm{~mm}$ triple resonance MAS probe, and ${ }^{15} \mathrm{~N}$ cross-polarization (CP) MAS NMR experiments ${ }^{20}$ were conducted on a $400 \mathrm{MHz}$ Avance III HD spectrometer using a $1.3 \mathrm{~mm}$ double resonance MAS probe. For the ${ }^{13} \mathrm{C} \mathrm{DP}$ experiment, excitation $\pi / 2$ pulses of $4.75 \mu$ s duration at a radio frequency (rf) power of $150 \mathrm{~W}$, corresponding to an rf field strength of $53 \mathrm{kHz}$, were used with a relaxation delay of $250 \mathrm{~s}$ to ensure complete relaxation of longitudinal spin magnetization. SPINAL-64 heteronuclear decoupling ${ }^{31}$ was applied during acquisition with a ${ }^{1} \mathrm{H}$ rf field of ca. $100 \mathrm{kHz}$. The number of accumulations was 16 at a spinning frequency of 20 $\mathrm{kHz}$, which is fast enough to remove spinning sidebands. ${ }^{15} \mathrm{~N}$ $\mathrm{CP}$ experiments were acquired at a spinning frequency of 30 $\mathrm{kHz}$ with a power of $60 \mathrm{~W}$ and 2048 scans for Glu/Uro-1:4 samples and 37600 scans for Glu/Uro-1:0.17 samples with a delay of $5 \mathrm{~s}$. The contact time was $7000 \mu$ s unless otherwise stated, and the proton $\pi / 2$ pulse width for cross-polarization 
Scheme 1. Synthetic Scheme of the Hydrothermal Carbonization for Different Amounts and Types of N-Functional Groups Using Glucose and Urotropine as Precursors

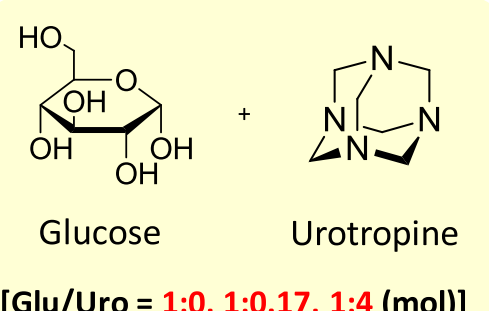

[Glu/Uro = 1:0, 1:0.17, 1:4 (mol)]
Hydrothermal carbonization

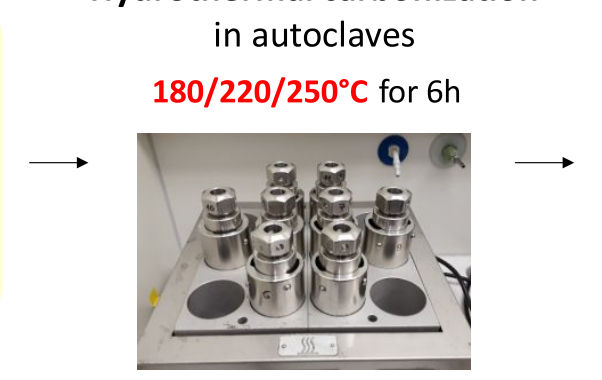

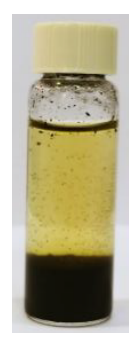
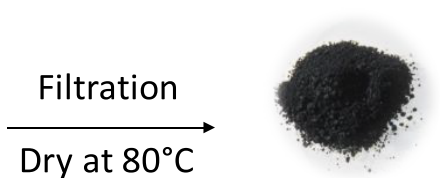

(N)-HTC was $1.3 \mu$ s. SPINAL-64 decoupling with a ${ }^{1} \mathrm{H}$ rf field of ca. 200 $\mathrm{kHz}$ was used during detection.

The (N)-HTC samples have been prepared as a slurry saturated with water for molecular dynamics study. Water volumes per surface area of powder in the NMR signal detection region are specified in Table S2. The NMR spectra of selected samples are shown in the Supporting Information (Figure S1). The detailed measurement method for the water volume per powder surface area is shown in Figure S2 and Table S2. The NMR sample tubes were sealed after filling. Before starting a temperature-dependent experiment, the samples were left to thermally equilibrate for at least $20 \mathrm{~min}$. All the diffusion and relaxation NMR experiments were performed on a Bruker Avance III HD 600 wide-bore spectrometer equipped with a gradient probe (Bruker PA BBO 600W2/S4 BB-H\&F-D-05 DIFF) with a shielded Zgradient coil connected to a GREAT60 current amplifier and capable of achieving pulsed field gradients up to $23.1 \mathrm{~T} / \mathrm{m}$. The gradient strength was first calibrated using the known diffusion coefficient of water, $2.30 \times 10^{-9} \mathrm{~m}^{2} / \mathrm{s}$ at $298 \mathrm{~K}^{32}$ For water diffusion measurements in the presence of $(\mathrm{N})$-HTC materials, pulsed-field-gradient (PFG) NMR was performed using a stimulated-echo (PGSTE) pulse sequence containing longitudinal eddy current delay (LED) and two spoil gradient pulses to minimize the effect of eddy currents and sequential pulse mismatch. A total of 16 magnetic field gradients of amplitude in the range between 0.03 and $0.08 \mathrm{~T} / \mathrm{m}$ with duration $\delta=1 \mathrm{~ms}$ were used. The mixing time $\Delta$ was fixed at $50 \mathrm{~ms}$, and the recycle delay was set to $10 \mathrm{~s}$. Diffusion coefficients were calculated based on the Stejskal-Tanner equation ${ }^{33}$ using the integrals of the ${ }^{1} \mathrm{H}$ spectra. If fits with a single diffusion component showed significant residuals, a twocomponent, biexponential least-squares fit to ${ }^{34}$

$$
I(g)=I_{0 \mathrm{~A}} \mathrm{e}^{-D_{\mathrm{A}} \gamma^{2} \delta^{2} g^{2}(\Delta-\delta / 3)}+I_{0 \mathrm{~B}} \mathrm{e}^{-D_{\mathrm{B}} \gamma^{2} \delta^{2} g^{2}(\Delta-\delta / 3)}
$$

was employed, where $D_{\mathrm{A}}$ and $D_{\mathrm{B}}$ are the diffusion coefficients and $I_{0 \mathrm{~A}}$ and $I_{\mathrm{OB}}$ are the signal intensities in the absence of PFGs, originating from two species A and B, respectively, $I$ is the observed signal intensity, $\gamma$ is the magnetogyric ratio of protons, and $g$ is the gradient strength. The width of the gradient pulses and the delay between midpoints of gradient pulses are denoted by $\delta$ and $\Delta$, respectively.

The NMR spin-lattice relaxation time constant, $T_{1}$, of protons in water in the presence of $(\mathrm{N})$-HTC was measured by using the standard inversion-recovery sequence $\left(180^{\circ}-\tau-\right.$ $90^{\circ}$-acquisition). ${ }^{35}$ Sixteen points with interpulse delay, $\tau$, from $\tau=0.1 \mathrm{~ms}$ to $\tau=15 \mathrm{~s}$ were collected. $T_{1}$ was determined by fitting the signal as a function of $\tau$ using

$$
I(\tau)=I_{0}\left(1-2 \mathrm{e}^{-\tau / T_{1}}\right)
$$

where $I_{0}$ is the signal from a sample at thermal equilibrium. In this experiment, $I(\tau)$ is proportional to $M_{z}$ at time $\tau$ immediately before the detection pulse, and $I_{0}$ is proportional to the equilibrium magnetization $M_{0}$. Fitting of a single $T_{1}$ component led to good fits for all of the recorded data sets.

Proton-deuterium (H/D) exchange was performed by using $\mathrm{D}_{2} \mathrm{O}$ to investigate which protons of the $\mathrm{N}$-functional groups of N-HTC would exchange with deuterium to confirm our water interaction model in N-HTC. An excess volume of $\mathrm{D}_{2} \mathrm{O}$ was added to Glu/Uro-1:4-250 and Glu/Uro-1:0.17-250, sonicated for $10 \mathrm{~min}$, and incubated for $24 \mathrm{~h}$ at $25^{\circ} \mathrm{C}$ for H/D exchange. Afterward, the supernatant was removed, and then the same procedure was repeated once more. Then the $\mathrm{N}$ HTC powder was dried in an oven at $80{ }^{\circ} \mathrm{C}$ for 2 days. ${ }^{15} \mathrm{~N}$ CPMAS NMR spectra of the H/D exchanged samples were acquired with the same parameter as in previous ${ }^{15} \mathrm{~N}$ CPMAS experiments, but two different contact times (7000 and 700 $\mu \mathrm{s})$ were used to compare the $\mathrm{CP}$ effect for the different $\mathrm{N}$ functional groups.

\section{RESULTS AND DISCUSSION}

Synthesis and Characterizations. In Scheme 1, the synthesis of N-HTC material using glucose and urotropine is illustrated. ${ }^{12}$ Glucose was used as a representative structure of biomass, and urotropine was used to introduce $\mathrm{N}$-functional groups to HTC. During the hydrothermal process, the acidcatalyzed dehydration of glucose directly forms 5hydroxymethylfurfural (HMF). ${ }^{36-38}$ HMF can be further decomposed into levulinic acid and formic acid in several steps. The decomposition process also involves a variety of side reactions leading to polymeric byproducts called humins. ${ }^{37,39}$

Several N-containing structural motifs can be formed through the reaction of the decomposition products of glucose with ammonia and formaldehyde, the degradation products of urotropine during the hydrothermal reaction. ${ }^{12}$ The chemical structure of the final N-HTC product is very complicated because the reactions between the decomposition products from both precursors can open up various possible reaction pathways. For example, the Maillard reactions, ${ }^{8,20}$ including amination, imination, Amadory rearrangement, cyclization, and Strecker degradation, and non-Maillard reactions, ${ }^{8,40}$ including Diels-Alder cycloaddition or imino-Diels-Alder reactions, occur simultaneously to form N-HTC. Because of this complexity, the introduction of nitrogen functional groups into the carbon network during hydrothermal treatment has not been fully understood yet. 
Glu:Uro = 1:0

$180^{\circ} \mathrm{C}$

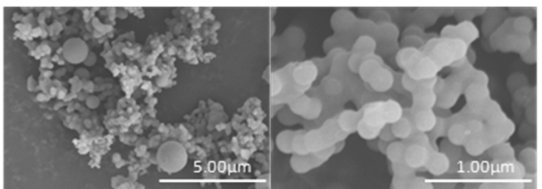

$220^{\circ} \mathrm{C}$

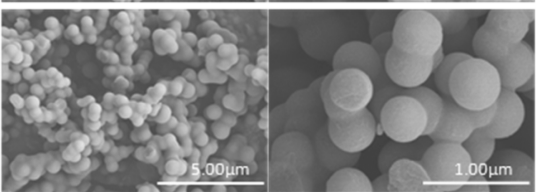

$250^{\circ} \mathrm{C}$

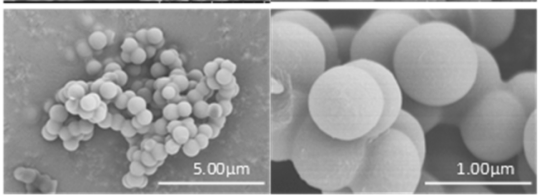

Glu:Uro = 1:0.17
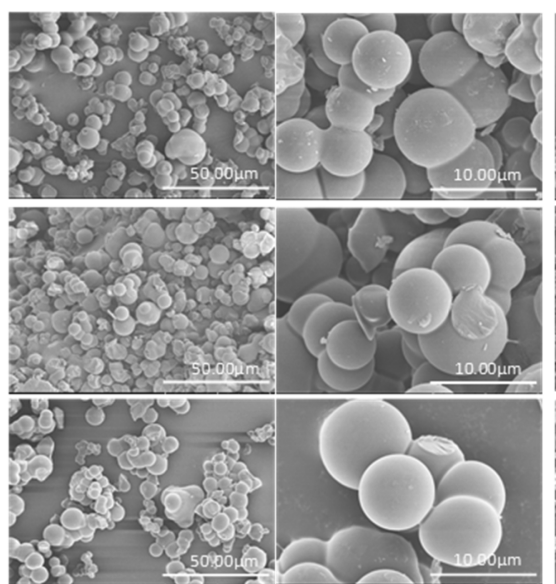
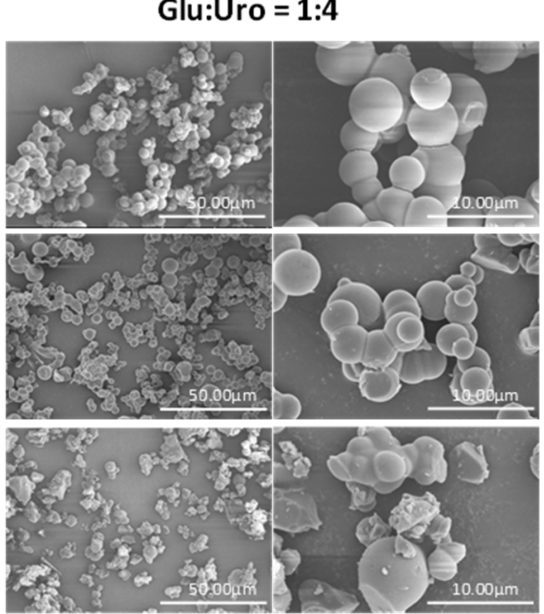

Figure 1. SEM images of (N)-HTCs with different precursor compositions and different synthesis temperatures. Note the 10 times smaller scale for Glu:Uro $=1: 0$.

To analyze the interactions between water and (N)-HTCs for different types and amounts of $\mathrm{N}$-functional groups, we prepared three different ratios Glu:Uro $=1: 0,1: 0.17$, and 1:4 at three different synthesis temperatures (180, 220, and 250 ${ }^{\circ} \mathrm{C}$ ) for the hydrothermal carbonization. Overall nine samples were analyzed to reveal the interaction properties of $(\mathrm{N})$ HTCs with water.

SEM was performed to confirm the surface morphology and particle size of the synthesized (N)-HTC materials. In Figure 1, SEM images show a homogeneous spherical morphology for N-free HTCs and Glu/Uro-1:0.17 samples. Glu/Uro-1:4 samples are slightly less uniform when synthesized at 180 and $220{ }^{\circ} \mathrm{C}$ but still show spherical morphology, while a heterogeneous morphology with irregular textures at a high synthesis temperature of $250{ }^{\circ} \mathrm{C}$ is observed. N-HTCs synthesized at 220 and $250{ }^{\circ} \mathrm{C}$ have a larger particle size than that of HTCs by a factor of around 10 and even larger at $180{ }^{\circ} \mathrm{C}$ by a factor of around 15 . In addition, the particle size increases as the synthesis temperature increases for HTC samples, while the change of particle size is not significant for Glu/Uro-1:0.17. For the Glu/Uro-1:4 samples, particle morphology related to synthesis temperature can again be clearly observed.

Heteroatom loading is crucial for changing the physicochemical properties of carbon materials. ${ }^{15}$ Elemental analysis of (N)-HTCs shows the carbon, hydrogen, nitrogen, and oxygen content dependence on the ratio of Glu/Uro and the synthesis temperature (Table 1). With a higher ratio of urotropine in the synthesis, a higher nitrogen content in the product is achieved, which means urotropine is taking part in the hydrothermal synthesis. When the synthesis temperature is elevated, the carbon content of the products increases and the oxygen content is reduced, indicating that more carbonization and more structural decomposition in the hydrothermal process are taking place.

The specific surface area of (N)-HTCs was investigated by nitrogen adsorption experiments and BET analysis (Table 1). The N-HTCs show very small surface areas below $1 \mathrm{~m}^{2} \mathrm{~g}^{-1}$, and the influence of synthesis temperature is relatively insignificant. Glu/Uro-1:4 samples provide a slightly larger surface area than Glu/Uro-1:0.17, particularly at low synthesis temperatures. On the other hand, the pure HTCs have a larger surface area of $2-8 \mathrm{~m}^{2} \mathrm{~g}^{-1}$, which is strongly influenced by the
Table 1. BET Surface Area and Elemental Analysis of (N)HTC Samples Synthesized at Different Temperatures

\begin{tabular}{clccccc}
\multicolumn{2}{c}{ samples } & & \multicolumn{5}{c}{ elemental analysis (at. \%) } \\
\cline { 5 - 7 } $\begin{array}{c}\text { synthesis } \\
\text { temp }\left({ }^{\circ} \mathrm{C}\right)\end{array}$ & \begin{tabular}{c} 
Glu/Uro \\
\cline { 5 - 7 } 180
\end{tabular} & $\begin{array}{c}\text { BET surface } \\
\text { area }\left(\mathrm{m}^{2} / \mathrm{g}\right)\end{array}$ & $\mathrm{C}$ & $\mathrm{H}$ & $\mathrm{N}$ & $\mathrm{O}$ \\
& $1: 4$ & 0.7 & 56.86 & 6.26 & 19.14 & 17.74 \\
& $1: 0.17$ & 0.4 & 61.57 & 5.49 & 6.35 & 26.59 \\
& $1: 0$ & 7.7 & 64.01 & 4.53 & N.D. & 31.46 \\
220 & $1: 4$ & 0.7 & 61.08 & 6.18 & 19.32 & 13.42 \\
& $1: 0.17$ & 0.5 & 66.22 & 5.43 & 7.29 & 21.06 \\
& $1: 0$ & 3.2 & 67.04 & 4.63 & N.D. & 28.33 \\
250 & $1: 4$ & 0.6 & 63.46 & 6.14 & 18.90 & 11.50 \\
& $1: 0.17$ & 0.5 & 69.63 & 5.37 & 7.58 & 17.42 \\
& $1: 0$ & 1.9 & 69.33 & 4.70 & N.D. & 25.97 \\
\hline
\end{tabular}

synthesis temperature, showing a smaller surface area at higher synthesis temperature. Only narrow hysteresis loops could be observed, which indicate capillary condensation effects of only a few pores. Nevertheless, no closed hysteresis loops could be obtained; therefore, no reliable statement about the presence or type of pores can be made (Figure S3). The BET surface areas for all the samples are consistent with the surface areas that can be estimated from the SEM images (Figure 1) by considering the $(\mathrm{N})$-HTC particles as spheres with a smooth surface.

To analyze the structural chaznges of N-HTC as a function of synthesis temperature, the ${ }^{13} \mathrm{C}$ DPMAS NMR and ${ }^{15} \mathrm{~N}$ CPMAS NMR spectra for Glu/Uro-1:4 and Glu/Uro-1:0.17 after hydrothermal treatment are compared in Figure 2. Increasing the synthesis temperature produces a hydrothermal carbon with a higher degree of aromatization. Evidence supporting this can be seen in both ${ }^{13} \mathrm{C}$ DPMAS and ${ }^{15} \mathrm{~N}$ CPMAS NMR spectra. In ${ }^{13} \mathrm{C}$ DPMAS, there is an increase in the relative intensity of the peaks at $100-150 \mathrm{ppm}$, which can be assigned to aromatic ring carbons. ${ }^{38}$ In addition, the peaks at $40-90 \mathrm{ppm}$ are decreased, which originate from aliphatic structures related to many different types of alkyl-ether and alkyl-amine groups. ${ }^{15} \mathrm{~N}$ CPMAS spectra show the increase in the relative intensity of the peaks at -40 to $-160 \mathrm{ppm}$ and at -180 to $-260 \mathrm{ppm}$, which can be assigned to nitrogen atoms of conjugated nitrogen functional groups such as pyrazine or pyridine and to pyrrole, respectively. The decrease of peaks at 
(a)

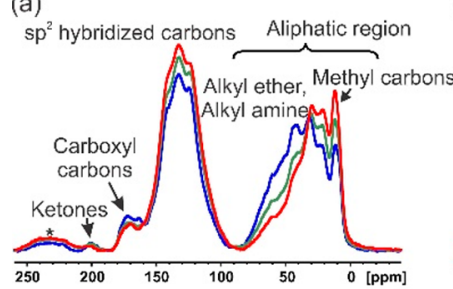

(c)

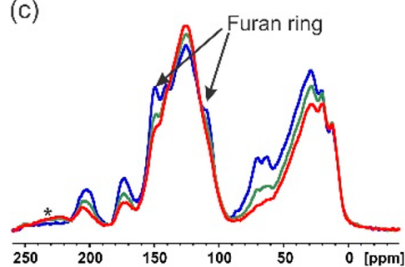

(b)

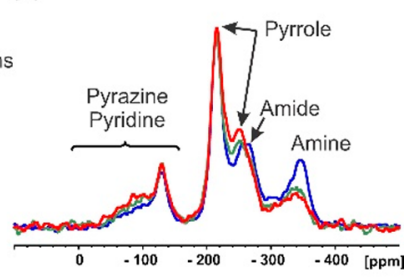

(d)

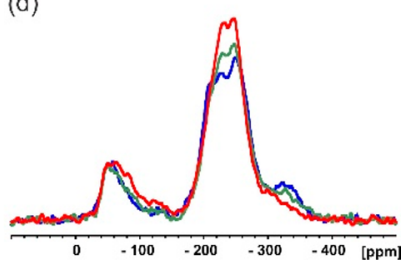

Figure 2. (a, c) ${ }^{13} \mathrm{C}$ DPMAS NMR spectra, and (b, d) ${ }^{15} \mathrm{~N}$ CPMAS NMR spectra of N-HTCs Glu/Uro-1:4 (a, b) and Glu/Uro-1:0.17 (c, d) obtained at different synthesis temperatures. Blue: $180{ }^{\circ} \mathrm{C}$; green: $220{ }^{\circ} \mathrm{C}$; red: $250{ }^{\circ} \mathrm{C}$. The intensity of the spectra was normalized by using the mass of each sample.

-260 to $-280 \mathrm{ppm}$ and -300 to $-380 \mathrm{ppm}$ also shows that the amide and amine related structures are reduced at high synthesis temperature. Additionally, increasing the temperature in N-HTC synthesis reduces the relative amount of carbonyl carbons represented at $160-220 \mathrm{ppm}$ in ${ }^{13} \mathrm{C}$ DPMAS, which is in accordance with a decrease in the oxygen content (Table 1 ). This phenomenon was already reported in N-free HTC synthesis. ${ }^{41}$ In the case of Glu/Uro-1:0.17 samples, the furan ring structures, which are not confirmed in Glu/Uro-1:4, are dramatically reduced with increasing synthesis temperature. Comparing the whole spectra, Glu/Uro-1:0.17 shows larger synthesis temperature-dependent structural chemical changes than Glu/Uro-1:4. Integrals for the different NMR resonance are summarized in Table S3.

Longitudinal NMR Relaxation of Water in (N)-HTC. The ${ }^{1} \mathrm{H} T_{1}$ relaxation time constant of water in the presence of (N)-HTC was measured to determine changes of the rotational correlation time of water due to the interaction between the functional groups of (N)-HTC and water molecules. The rotational correlation time constant, $\tau_{\mathcal{c}}$ is the average time it takes for a molecule to rotate by $1 \mathrm{rad}$. In general, it is well recognized that an increase of $\tau_{\mathrm{c}}$ caused by restricted molecular motions leads to a reduction of $T_{1}$ for small molecules such as $\mathrm{H}_{2} \mathrm{O}{ }^{42}$

Figure S4 shows the results of the inversion-recovery experiment of $(\mathrm{N})$-HTC plotted as the natural logarithm of $\left(I_{0}\right.$ $-I(\tau)) /\left(2 I_{0}\right)$, which is equal to $\ln \left\{\left(M_{0}-M_{z}(\tau) / 2 M_{0}\right\}\right.$, vs $\tau$. All experiments exhibited a straight line, implying a monoexponential decay of $M_{z}$ with a slope equal to $-1 / T_{1}$. Therefore, $T_{1}$ either is homogeneous and independent of the water location or is averaged out over different water locations in the presence of (N)-HTC by the motion of water molecules, indicating that water samples the complete porous environment within a time given by $T_{1}$. Because the $T_{1}$ values of water in the presence of $(\mathrm{N})-\mathrm{HTC}$, shown in Figure 3a, differ from $T_{1}$ of free water, the latter case is more likely, meaning that exchange rates for water molecules between different surface locations in the sample are shorter than $T_{1}$ (fast exchange limit). ${ }^{43}$ This is consistent with a previous report that water showed a single $T_{1}$ component for single-walled carbon nanotubes (SWCNT). ${ }^{44}$
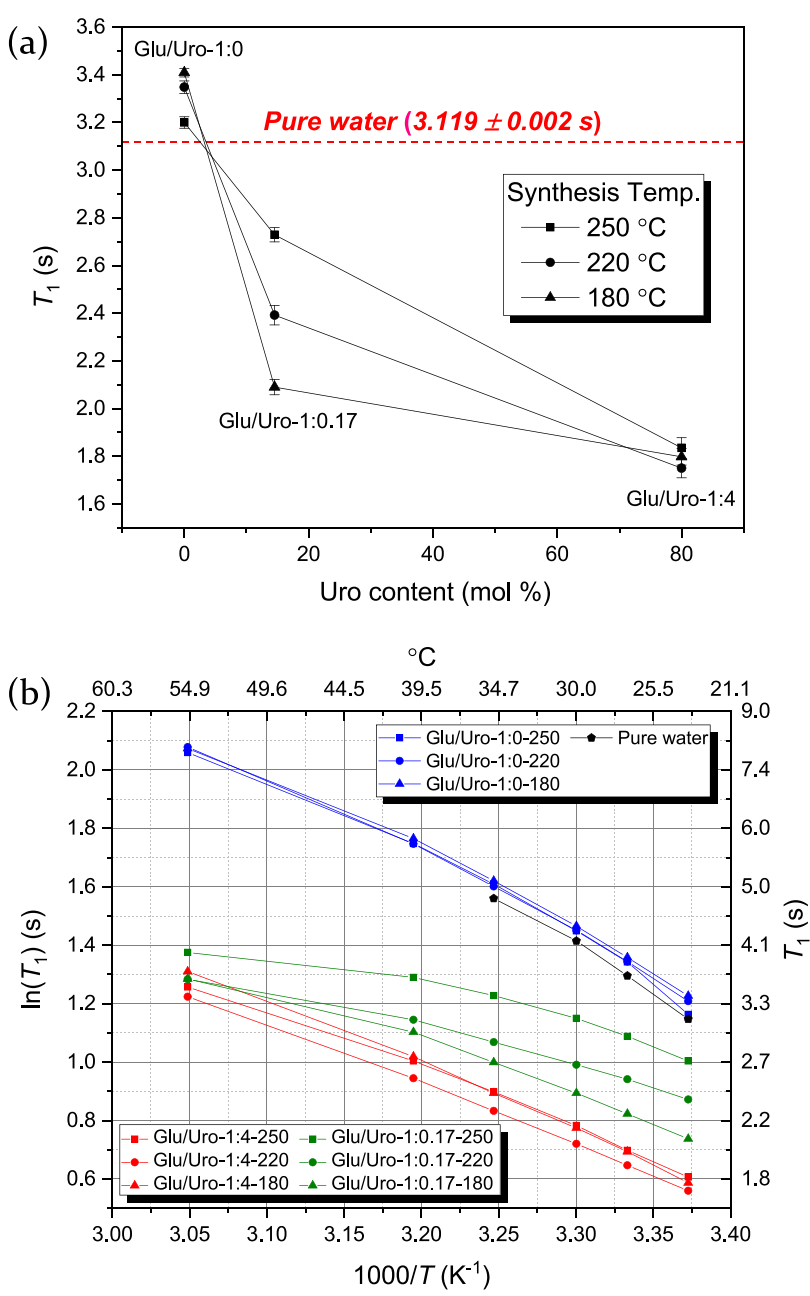

Figure 3. (a) Spin-lattice relaxation time $T_{1}$ values of water in the presence of (N)-HTC at $23.5{ }^{\circ} \mathrm{C}$. Red dashed line: $T_{1}$ value of pure water without (N)-HTC material. (b) Arrhenius plot of the $T_{1}$ relaxation time for pure water and water in the presence of $(\mathrm{N})$-HTC.

In the fast exchange limit, the observed relaxation rate $R_{1}=$ $1 / T_{1}$ is the weighted mean of the relaxation rates experienced by the molecules

$$
\frac{1}{T_{1}}=\frac{p_{\text {free }}}{T_{1}^{(\text {free })}}+\sum_{i=1}^{N} \frac{p_{i}}{T_{1}^{(i)}}
$$

where a water molecule is found with probability $P_{\text {free }}$ in an environment unaffected by $(\mathrm{N})$-HTC and with probability $p_{i}$ in one of $N$ different $(\mathrm{N})$-HTC environments. $T_{1}^{(\text {free })}$ is the free water relaxation time constant, and $T_{1}^{(i)}$ is the relaxation time constant in the $i$ th $(\mathrm{N})$-HTC environment.

The $T_{1}$ values of Glu/Uro-1:0 samples are longer than $T_{1}$ of pure water. For fluids interacting with a porous medium, there can be a number of different relaxation contributions, in particular surface relaxation ${ }^{45}$ and relaxation due to diffusion in a nonuniform magnetic field caused by susceptibility differences near an interface. ${ }^{46}$ Assuming that the correlation time constant, $\tau_{\mathcal{c}}$ of the fluid motion would be unaffected by the porous medium, all these contributions cause an increase of the relaxation rate $1 / T_{1}^{(i)}$ compared to the free fluid, $1 / T_{1}^{(\text {free })}$. The observation for water in HTC that $T_{1}^{(i)}>T_{1}^{(\text {free })}$ is therefore significant insofar as it implies a decrease of $\tau_{c}$ for the relaxation dominating motion. ${ }^{47}$ Such a decrease of $\tau_{\mathrm{c}}$ may be 
the result of the hydrophobic properties of HTC. Faster water dynamics could be achieved by increasing the hydrophobicity of the materials. ${ }^{44}$ Even though HTC shows a greater incorporation of oxygen-containing functional groups than conventional carbon materials, the oxygenated functionalities in HTC materials appear to play a minor role in the interaction with water.

Water in N-HTC has a shorter $T_{1}$ than in HTC, which is further decreasing with increasing $\mathrm{N}$ content. While this acceleration observed for N-HTC could have a number of reasons, neither surface relaxivity in the absence of radical species nor diffusion in a nonuniform magnetic field is expected to show such a pronounced difference compared to HTC. Possible reasons could be an increase of $\tau_{c}$, but the existence of additional fluid environments with either increased surface relaxivity, considerable susceptibility gradients, or altered correlation times would be possible. An increase of $\tau_{\mathrm{c}}$ could be rationalized by a stronger interaction of $\mathrm{N}$-functional groups with water molecules compared to $\mathrm{O}$-functional groups in HTC. On the other hand, especially surface relaxivity is dependent on the surface-to-volume ratio and therefore on the pore size distribution. ${ }^{48}$ However, as shown in the BET results (Table 1), N-HTCs show a very small surface area with negligible intraparticle pores that is only weakly synthesis temperature and $\mathrm{N}$ content dependent. This would imply that the difference of the water relaxivity in N-HTC could be interpreted as an effect of the presence of $\mathrm{N}$-functional groups, with a minor pore size contribution.

Especially for the Glu/Uro-1:0.17 samples, $T_{1}$ values are synthesis temperature dependent. The higher the synthesis temperature, the longer $T_{1}$. This could be explained by $\mathrm{N}$ functional groups changing into more conjugated groups, which are less hydrophilic, at higher synthesis temperature. On the other hand, in the case of Glu/Uro-1:0, no significant changes were observed with synthesis temperature. This indicates that the reorientational rotation of water cannot be changed significantly by the synthesis temperature for HTC, which may be explained by the high hydrophobicity already at low synthesis temperature.

Figure $3 \mathrm{~b}$ shows the behavior of the $T_{1}$ relaxation times measured at six different sample temperatures in an Arrhenius plot. The water in HTC always shows a longer $T_{1}$ than pure water. In addition, the behavior of $T_{1}$ as a function of temperature is quite similar for pure water and for water in HTC. This indicates that the activation energy for reorientational molecular rotation is very similar between the pure water and the water in HTC material, which also means that HTC only weakly interacts with water.

$T_{1}$ of water in N-HTC samples is shorter than in HTC at the same experimental temperature. The difference becomes larger as the temperature increases, with $T_{1}$ of water in N-HTC being less temperature dependent, which could be explained by the interaction with $\mathrm{N}$-functional groups. Especially when looking at the Glu/Uro-1:0.17 samples synthesized at different temperatures, the difference in $T_{1}$ relaxation time decreases as the experimental temperature increases. This indicates that the activation energy for reorientational molecular rotation is synthesis temperature dependent.

Effect of the Functional Groups in (N)-HTC on LongRange Water Translational Motion. The self-diffusion of water molecules over length scales on the order of $\mu \mathrm{m}$ was measured by using PFG diffusion NMR. All experiments were performed in water-saturated $(\mathrm{N})$-HTC samples at $23.5^{\circ} \mathrm{C}$. In
Figure 4, diffusion results are plotted as the natural logarithm of the normalized signal intensity vs the squared gradient

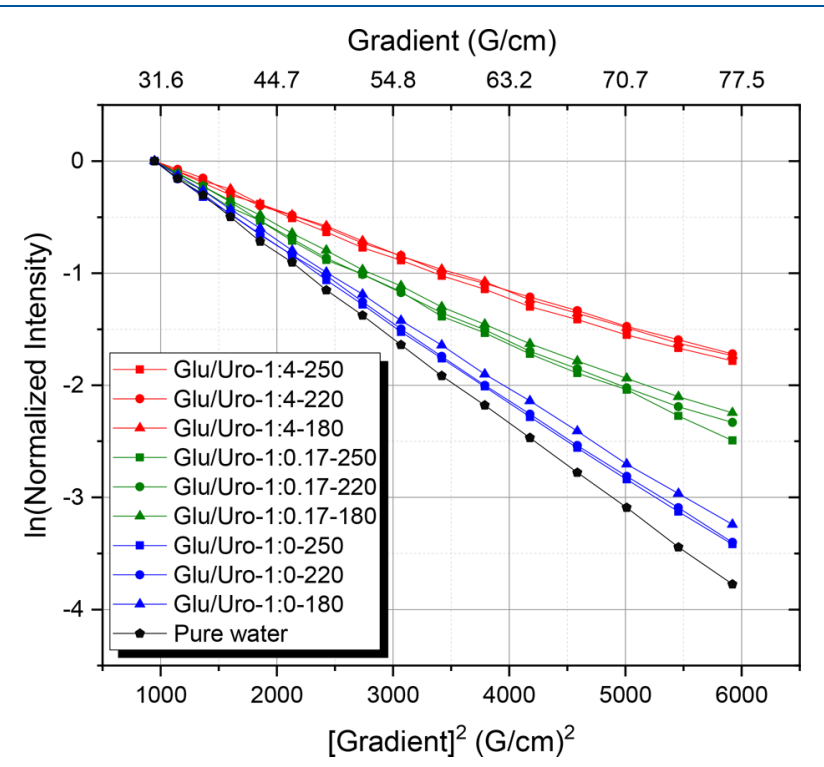

Figure 4. Plot of log intensity vs squared gradient strength for selfdiffusion of water in the presence of $(\mathrm{N})$-HTC materials made with different Glu/Uro ratios and at different synthesis temperatures. Red, green, and blue lines represent the different Glu/Uro ratios 1:4, 1:0.17, and 1:0, respectively. The black line represents pure water.

amplitude. For the Glu/Uro-1:0 samples these curves decay linearly, while N-HTC samples show a stretched decay. A straight line in this plot is interpreted as a single component of water translational motion, and the slope becomes an effective diffusion constant according to the Stejskal-Tanner equation. As in the relaxation experiments, the suitability of a linear fit on the log scale for the HTC sample does not imply the existence of a single diffusion constant only. Instead, diffusion in the limit of fast exchange over a time scale given by the mixing time $\Delta$ of the diffusion experiment (here $\Delta=50 \mathrm{~ms}$ ) would equally result in such a decay. On the other hand, the data for the N-HTC sample do not coincide with a straight line. This is interpreted as being caused by water in two different reservoirs in the intermediate or slow exchange limit, meaning that no or incomplete mixing of the reservoirs is achieved during the experiment. The PGSTE NMR experiment is sensitive to translational motion over length scales of micrometers; therefore, these results indicate not only that the two reservoirs do not mix or mix slowly but also that water moves over at least hundreds of nanometers within each of the reservoirs without exchanging. ${ }^{49}$ Time-dependent diffusion NMR experiments, ${ }^{50,51}$ recorded with different $\Delta$, are also consistent with two coupled water reservoirs in the slow exchange limit in $\mathrm{N}$ HTC, which is not seen in HTC (Figure S5).

The fitted effective diffusion coefficients are shown in Table 2. For N-HTC, biexponential fits using eq 1 were performed, while single-exponential fits in the case of HTC and pure water were used. In all (N)-HTC samples, the translational motion of water is slower than that of pure water, which is probably a combined effect of geometric restrictions for the water motion $^{50}$ on the one hand and interactions between the functional groups and the water molecules ${ }^{52}$ on the other. Glu/Uro-1:4 samples with the largest amount of $\mathrm{N}$-functional groups show a slower translational motion than other samples, 
Table 2. PFG NMR Detected Diffusion Coefficients of Water at $23.5{ }^{\circ} \mathrm{C}$ in the Presence of (N)-HTC Materials Made with Different Glu/Uro Ratios and at Different Synthesis Temperatures ${ }^{a}$

\begin{tabular}{|c|c|c|c|c|c|}
\hline \multicolumn{2}{|c|}{ sample } & \multirow[b]{2}{*}{$\begin{array}{l}\text { water volume per surface area of powder } \\
\text { in the detection region }\left(\mathrm{mL} / \mathrm{m}^{2}\right)\end{array}$} & \multirow[b]{2}{*}{ fast diff coeff $\left(\mathrm{m}^{2} / \mathrm{s}\right)$} & \multirow[b]{2}{*}{ slow diff coeff $\left(\mathrm{m}^{2} / \mathrm{s}\right)$ [relative amount] } & \multirow[b]{2}{*}{ fit model } \\
\hline $\begin{array}{l}\text { synthesis } \\
\text { temp }\left({ }^{\circ} \mathrm{C}\right)\end{array}$ & Glu/Uro & & & & \\
\hline \multirow[t]{3}{*}{180} & $1: 4$ & 2.1 & $(1.30 \pm 0.01) \times 10^{-9}$ & $(1.78 \pm 0.17) \times 10^{-10}[8.6 \%]$ & double \\
\hline & $1: 0.17$ & 3.2 & $(1.70 \pm 0.01) \times 10^{-9}$ & $(1.85 \pm 0.16) \times 10^{-10}[5.1 \%]$ & double \\
\hline & $1: 0$ & 0.9 & $(1.89 \pm 0.01) \times 10^{-9}$ & & single \\
\hline \multirow[t]{3}{*}{220} & $1: 4$ & 1.7 & $(1.39 \pm 0.01) \times 10^{-9}$ & $(1.29 \pm 0.24) \times 10^{-10}[9.2 \%]$ & double \\
\hline & $1: 0.17$ & 2.8 & $(1.84 \pm 0.01) \times 10^{-9}$ & $(2.29 \pm 0.29) \times 10^{-10}[6.1 \%]$ & double \\
\hline & $1: 0$ & 1.9 & $(1.97 \pm 0.01) \times 10^{-9}$ & & single \\
\hline \multirow[t]{3}{*}{250} & $1: 4$ & 2.1 & $(1.42 \pm 0.01) \times 10^{-9}$ & $(1.55 \pm 0.17) \times 10^{-10}[8.7 \%]$ & double \\
\hline & $1: 0.17$ & 1.9 & $(1.83 \pm 0.01) \times 10^{-9}$ & $(3.12 \pm 0.40) \times 10^{-10}[5.8 \%]$ & double \\
\hline & $1: 0$ & 2.9 & $(1.99 \pm 0.01) \times 10^{-9}$ & & single \\
\hline pure water & & & $(2.20 \pm 0.01) \times 10^{-9}$ & & single \\
\hline
\end{tabular}

${ }^{a_{T}}$ The diffusion coefficient of pure water under identical conditions is provided as a reference point. The relative signal amplitude of the slow diffusion component is given in square brackets. This provides only a qualitative lower boundary since signal weighting due to different spin-spin relaxation time constants $\left(T_{2}\right)$ is not compensated. Errors are estimated from the residuals of the fit.

suggesting that these samples interact more strongly with water. Even in the case of HTC with a small water volume per powder surface area, the water dynamics are faster than that of $\mathrm{N}$-HTC. Therefore, the difference in the water dynamics is due to the effect of $\mathrm{N}$-functional groups rather than the difference in the amount of water.

No dramatic changes were observed for different synthesis temperatures. This is to some degree in contrast to the $T_{1}$ data that showed a pronounced synthesis temperature dependence, particularly for the Glu/Uro-1:0.17 sample. It indicates that the increased motional correlation time identified in the $T_{1}$ experiments is not the only reason for the slower long-range motion of water. Instead, diffusion in particular of the slowmoving component in N-HTC may be governed by additional physical constrictions such as confined space and tortuosity effects, i.e., the absence of direct paths that would allow motion over micrometer length scales without detours. Such physical effects could also explain the slow exchange between the reservoirs. On the other hand, the void space between the particles is negligible as the second reservoir. In HTC, where the smaller particle diameter would suggest smaller voids and hence a more pronounced effect on the water mobility, no additional slow diffusion component is observed. Given the low BET surface and the absence of intraparticle pores, this implies that water responsible for the slow diffusion is adsorbed inside the N-HTC material in an environment not accessible to the gas used for the BET experiments. Considering that the (N)-HTC have an amorphous network (Figure S6), the confined space where water molecules exist might be generated due to the softening influence of water interacting with $\mathrm{N}$-functional groups on this network in $\mathrm{N}$ HTC. A shortening of the ${ }^{13} \mathrm{C} T_{1}$ relaxation time in the $\mathrm{N}$ HTC carbon network upon addition of water is an indication for such a softening behavior (Figure S7).

The fact that two different effective diffusion constants are apparent for the N-HTC samples means that water resides in two different environments without complete mixing for a duration given by $\Delta$ and that water displacement over hundreds of nanometers occurs without exchange. In addition, the faster of the two diffusion coefficients is lower than the selfdiffusion coefficient of free water, indicating some additional hindrance either due to tortuosity effects or due to water interaction with the particle surface and fast exchange on the
NMR time scale. Considering the low BET surface in particular for the N-HTC material (Table 1), the latter option appears more likely. Hence, in analogy to the previous result for the longitudinal relaxation, the effective diffusion coefficient can be written as

$$
D_{\text {eff }}=p_{\text {free }} D_{0}^{\left(\mathrm{H}_{2} \mathrm{O}\right)}+\sum_{i=1}^{N} p_{i} D_{\text {eff }}^{(i)}
$$

where $D_{0}^{\left(\mathrm{H}_{2} \mathrm{O}\right)}$ represents the self-diffusion coefficient of free water and $D_{\text {eff }}^{(i)}$ is the effective diffusion coefficient in environment $i$, which experiences fast exchange with the free water reservoir. Notice that eq 4 applies independently to both $D_{\mathrm{A}}$ and $D_{\mathrm{B}}$ in eq 1 . A stronger reduction of $D_{\text {eff }}$ could be caused by lower $D_{\text {eff }}^{(i)}$ or by a higher $p_{i}$. In this system, both possibilities are conceivable. Nevertheless, the lower BET surface for the NHTC samples (Table 1) in combination with slow moving water in N-HTC being distingushable from fast moving water (Table 2) may provide a hint that the fast $D_{\text {eff }}$ represents an average value of the weighted diffusion coefficients between bulk water and water in the hydration shell formed on the surface of N-HTC. Simulations suggest the exchange rate between the bulk water and the hydration shell is expected to be on the order of pico- to nanoseconds; ${ }^{53}$ hence, these two components cannot be distinguished on the diffusion NMR time scale (milliseconds). Therefore, the reduced fast diffusion coefficient of N-HTC indicates either a more complete, a thicker, or at least a differently structured hydration shell than for HTC.

Temperature-dependent diffusion NMR experiments were performed at six different sample temperatures from 23.5 to 55 ${ }^{\circ} \mathrm{C}$ to investigate the stability of the water with the slow diffusion coefficient (Figure S8). As the experiment temperature is raised, the chemical exchange with bulk water molecules is expected to increase. Regardless, N-HTC samples still show the two-component decay until $55^{\circ} \mathrm{C}$, while HTC samples show a single-component decay throughout.

The observation of distinguishable components in PFG diffusion experiments that are not exchange averaged during a mixing time as long as $\Delta=50 \mathrm{~ms}$ even at elevated temperatures is fairly significant. The slow diffusion constants in Table 2 suggest a motion of the water without exchange with the main reservoir on length scales comparable with the 
diameter of the N-HTC particles (Figure 1). It is improbable that such a motion occurs solely on the N-HTC surface or the void volumes between particles. Instead, internal water in the $\mathrm{N}-\mathrm{HTC}$ particles that can more or less freely move through the whole grain may explain such an observation. Alternatively, motion close to the surface due to particle depth-dependent differences in the softening effect of the amorphous network by water may also lead to similar results.

Identification of Internal Water in N-HTC with Deuterium Exchanged Samples. To investigate whether water leads to a swelling of the whole N-HTC particles or whether water adsorption in near-surface layers is more probable, deuterium exchange experiments were performed. Taking into account that the protons of $\mathrm{N}$-functional groups such as amines and amides can exchange well with deuterium, immersing previously water treated N-HTC in $\mathrm{D}_{2} \mathrm{O}$ for a sufficiently long time should cause an exchange of these protons with deuterium. If $\mathrm{D}_{2} \mathrm{O}$ was present in the entire volume of an N-HTC particle, most of the protons of the amines and amides would get replaced by deuterium, and the signals of these functional groups in a ${ }^{1} \mathrm{H}-{ }^{15} \mathrm{~N}$ CPMAS spectrum would be expected to decrease drastically. However, as can be seen in Figure 5, even though the overall peak
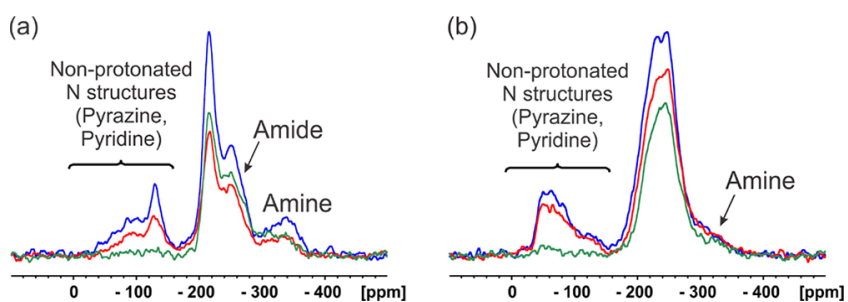

Figure 5. ${ }^{15} \mathrm{~N}$ CPMAS NMR spectra of (a) Glu/Uro-1:4-250 and (b) Glu/Uro-1:0.17-250, obtained before and after deuterium exchange as a function of contact time. Blue: before deuterium exchange (contact time $=7000 \mu \mathrm{s})$; red: after deuterium exchange (contact time $=7000$ $\mu \mathrm{s})$; green: after deuterium exchange (contact time $=700 \mu \mathrm{s})$. The intensity of the spectra was normalized by using the mass of each sample.

intensity is reduced, the amide- and amine-related peaks have similar amplitudes as in the spectrum before $\mathrm{D}_{2} \mathrm{O}$ treatment when using the same contact time $(7000 \mu \mathrm{s})$.

To verify whether this result was caused by the CP effect of long-range protons due to the long contact time, ${ }^{1} \mathrm{H}-{ }^{15} \mathrm{~N}$ CPMAS was also conducted using a short contact time $(700$ $\mu \mathrm{s})$. The amide and amine peaks are still observed at the short contact time unlike nonprotonated pyridine and pyrazine, which is a clear indication that $\mathrm{D}_{2} \mathrm{O}$ did not exchange protons of amines and amides to deuterium in the whole of the particles. Therefore, $\mathrm{D}_{2} \mathrm{O}$ cannot be present in the entire volume of an N-HTC particle, but likely just near the surface. Comparing the spectrum of Glu/Uro-1:4 and Glu/Uro-1:0.17, the peak intensities of Glu/Uro-1:4 were more strongly decreased after $\mathrm{D}_{2} \mathrm{O}$ treatment, which means that a larger fraction of the particle volume contains internal water or that a larger fraction of the amides and amines are located near the particle surface for Glu/Uro-1:4 than for Glu/Uro-1:0.17.

The additional results of ${ }^{1} \mathrm{H}$ HR-MAS (high resolutionmagic angle spinning) NMR after mixing a small amount of $\mathrm{D}_{2} \mathrm{O}(30 \mu \mathrm{L})$ with $(\mathrm{N})$-HTC (Figures $\mathrm{S} 9$ and $\mathrm{S} 10$ ) are in accordance with the results of ${ }^{15} \mathrm{~N}$ CPMAS with deuterium exchanged samples (Figure 5). HTC (Glu/Uro-1:0-180) shows only one water signal, while N-HTC (Glu/Uro-1:4180) shows two resonances containing a broad downfield shifted signal that can be assigned to the internal water in the ${ }^{1} \mathrm{H}$ HR-MAS spectrum.

In addition, optical microscopy was performed to confirm particle growth as a function of time after addition of water (Figures S11 and S12). The microscopic images show a particle growth as water enters through the particle surface for N-HTC (Glu/Uro-1:4-220), whereas this effect could not be confirmed for HTC (Glu/Uro-1:0-250).

Model for Interaction of Water with (N)-HTC. The information from the different experiments can be combined to a model that describes the interaction of water with $(\mathrm{N})$ HTC. The water motion was more restricted in N-HTC than in N-free HTC despite a lower BET surface area in the former, thereby suggesting that the N-HTC interacts more strongly with water molecules via the $\mathrm{N}$-functional groups than via the $\mathrm{O}$-functional groups in (N)-HTC. As a result, N-HTC forms a more complete or a thicker hydration shell than HTC.

While the surface area accessible in the BET experiment largely corresponds to the surface of the (N)-HTC particles, water is selectively able to percolate beneath the surface of $\mathrm{N}$ HTC. It appears that by interacting with $\mathrm{N}$-functional groups on the surface of N-HTC water opens up channels or "bottlenecks" that facilitate a slow exchange of water molecules through the N-HTC particle surface. ${ }^{54}$ The percolated water molecules are then able to adsorb into N-HTC particles near their surface and thereby establish a subsurface physicochemical structural region or reservoir. This is probably made possible by a softening of the amorphous structure of the $\mathrm{N}$ HTC caused by interactions of water molecules with nearsurface $\mathrm{N}$-functional groups. However, maybe due to structural rigidity or a radially dependent physicochemical structural accessibility of the N-HTC framework, such a softening is restricted to near-surface regions and does not lead to swelling of the whole particles.

The subsurface water access appears to span the whole particle surface, allowing for long-range motion of water molecules within this reservoir. Exchange with the surface water is slow even at elevated temperatures. The exchange time constant can be estimated to be slower than the mixing time in the PFG NMR experiment, yet faster than $T_{1}$, where only a single component was observed, since it is unlikely that longitudinal relaxation is equal in both environments. Therefore, a rough guess for the exchange time constant would place it to be on the order of hundreds of milliseconds.

In contrast, although N-free HTC has an amorphous structure similar to N-HTC (Figure S6), no such environment could be detected for HTC, indicating that the O-functional groups do not show the same kind of softening effect upon contact with water like the N-functional groups in N-HTC.

Based on the relationship between structure and water interaction, the key factors to enhance the interaction with water are $\mathrm{N}$-functional groups rather than $\mathrm{O}$-functional group or the pore size of (N)-HTC. We further assume that the water interaction with the N-HTC strongly depends on the $\mathrm{p} K_{\mathrm{a}}$ values of the $\mathrm{N}$-functional groups. Especially primary aliphatic amines are supposed to be protonated under the employed conditions and cause local water interactions. Therefore, this effect will probably depend on the $\mathrm{pH}$ value of the medium and will be part of future studies. Based on the analysis mentioned above, a schematic drawing of water interaction in the presence of N-HTC and HTC is shown in Figure 6. 

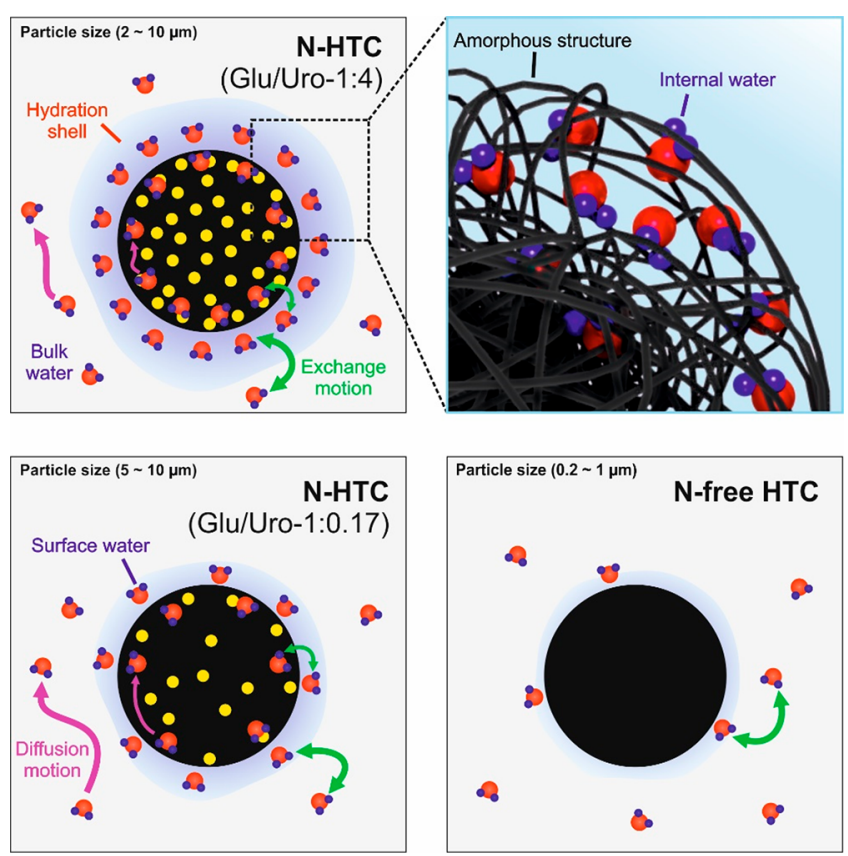

Figure 6. Schematic diagram of water interaction with N-HTC and HTC. (top left) N-HTC particle with high N content. Water motion on the surface is hindered, yet exchange with bulk water is fast on time scales probed by $T_{1}$ relaxation and PFG diffusion NMR experiments. In addition, a subsurface reservoir of water percolating into the particle is formed (see magnification in the top right panel). These water molecules do not penetrate the whole particle volume; swelling occurs only close to the particle surface. (bottom left) At reduced $\mathrm{N}$ content, the amount of water percolating into the particle is lower and water motion is somewhat slower. (bottom right) In the $\mathrm{N}$-free HTC, no water is percolating into the particle. The effective diffusion coefficient of water, which averages to a single value in the case of fast exchange between bulk-like water and near-surface water, is higher than for the respective reservoir in N-HTC but reduced compared to free water. Yellow dots represent the nitrogen functional groups. Thicker lines indicate faster processes, and thinner lines represent a higher barrier for water motion.

\section{CONCLUSIONS}

A strategy was presented to control the interaction of water with N-HTC by adjusting synthesis temperature as well as the ratio of $\mathrm{N}$-free to $\mathrm{N}$-containing precursors. The type and amount of $\mathrm{N}$-functional groups can be controlled by the precursor ratio, and the amount of the conjugated $\mathrm{N}$-functional groups can be tuned precisely via HTC synthesis temperature. These are key parameters that determine the interaction of (N)-HTC materials with water. Water motion was more restricted in N-HTC than in N-free HTC, thereby suggesting that N-HTC interacts with water molecules via the Nfunctional groups. The main difference between HTC and conventional carbon materials, such as graphene or carbon nanotubes, is the much greater incorporation of oxygencontaining functional groups. Nevertheless, the oxygenated functionalities in HTC play a rather minor role in the interaction with water. After nitrogen functionalization on HTC, the water interaction is increased, resulting in more complete or thicker hydration shells and internal water. Furthermore, although HTC shows a larger surface area than the N-HTC samples, the faster water dynamics in HTC proves that the $\mathrm{N}$-functional groups have a greater effect on the dynamics of the water than the pore size.
Understanding the water interaction with the N-HTC surface is expected to help tailoring the surface structure, especially when applying N-HTC as an electrode for water splitting. Further studies are ongoing to reveal the relationship between the hydration shell on N-HTC and the performance as an electrocatalyst for oxygen evolution reactions.

\section{ASSOCIATED CONTENT}

\section{Supporting Information}

The Supporting Information is available free of charge on the ACS Publications website at DOI: 10.1021/acs.jpcc.9b05323.

Description of hydrothermal synthetic recipe and yield; relative peak areas [\%] in ${ }^{13} \mathrm{C}$ DPMAS and ${ }^{15} \mathrm{~N}$ CPMAS NMR spectra of (N)-HTC; ${ }^{1} \mathrm{H}$ NMR spectrum of water in the presence of $(\mathrm{N})-\mathrm{HTC}$; the measurement method for the volume fraction of water; the calculation table for water volume per power surface area; nitrogen adsorption/desorption isotherms of (N)-HTC; plot of water $T_{1}$ relaxation in $(\mathrm{N})$-HTC; time-dependent water diffusion NMR results; HRTEM images of (N)-HTC; ${ }^{13} \mathrm{C}$ signal buildup curve of saturation recovery NMR experiment for ${ }^{13} \mathrm{C} /{ }^{15} \mathrm{~N}$-labeled Glu/Uro-1:4-220; plot of water self-diffusion in (N)-HTC depending on the experiment temperature; ${ }^{1} \mathrm{H}$ and ${ }^{1} \mathrm{H}$ 1D CPMG-detected MAS spectrum after mixing water; optical microscopic images of (N)-HTC after the addition of water (PDF)

\section{AUTHOR INFORMATION}

\section{Corresponding Authors}

*E-mail philipp.schleker@cec.mpg.de.

*E-mail saskia.heumann@cec.mpg.de.

ORCID $\odot$

P. Philipp M. Schleker: 0000-0002-6366-0693

Saskia Heumann: 0000-0003-3594-6392

Notes

The authors declare no competing financial interest.

\section{ACKNOWLEDGMENTS}

The authors thank Dr. Christoph Scheurer from TU Munich for helpful discussions regarding possible morphologies of the (N)-HTC. Furthermore, we thank Jungwon Park from LG Chem for SEM and TEM measurements. We are grateful to Sven Jovanovic for assistance with the optical microscopy. We also thank Birgit Deckers (Grafikbüro in MPI CEC) for drawing the schematic diagram of water interaction. Financial support from the Max Planck Society and funding by the German Federal Ministry of Education and Research (BMBF project SABLE, grant 03EK3543) for the $600 \mathrm{MHz}$ NMR spectrometer are gratefully acknowledged as well as for the BMBF MANGAN project (grant 03SF0509). H.P. thanks the dispatching for a doctoral degree by LG Chem.

\section{REFERENCES}

(1) Kinoshita, K. Carbon: Electrochemical and Physicochemical Properties; Wiley-Interscience: 1988.

(2) Dai, L.; Chang, D. W.; Baek, J.-B.; Lu, W. Carbon Nanomaterials for Advanced Energy Conversion and Storage. Small 2012, 8 (8), $1130-1166$

(3) Aricò, A. S.; Bruce, P.; Scrosati, B.; Tarascon, J.-M.; Van Schalkwijk, W. Nanostructured Materials for Advanced Energy Conversion and Storage Devices. Nat. Mater. 2005, 4, 366-377. 
(4) Choma, J.; Marszewski, M.; Osuchowski, L.; Jagiello, J.; Dziura, A.; Jaroniec, M. Adsorption Properties of Activated Carbons Prepared from Waste CDs and DVDs. ACS Sustainable Chem. Eng. 2015, 3 (4), 733-742.

(5) Guo, Y. G.; Hu, J. S.; Wan, L. J. Nanostructured Materials for Electrochemical Energy Conversion and Storage Devices. Adv. Mater. 2008, 20 (15), 2877-2887.

(6) Rolison, D. R.; Long, J. W.; Lytle, J. C.; Fischer, A. E.; Rhodes, C. P.; McEvoy, T. M.; Bourg, M. E.; Lubers, A. M. Multifunctional 3D Nanoarchitectures for Energy Storage and Conversion. Chem. Soc. Rev. 2009, 38 (1), 226-252.

(7) Titirici, M.-M.; Antonietti, M. Chemistry and Materials Options of Sustainable Carbon Materials Made by Hydrothermal Carbonization. Chem. Soc. Rev. 2010, 39 (1), 103-116.

(8) Titirici, M.-M. Sustainable Carbon Materials from Hydrothermal Processes, 1st ed.; John Wiley \& Sons, Ltd.: 2013.

(9) Zhao, Y.; Nakamura, R.; Kamiya, K.; Nakanishi, S.; Hashimoto, K. Nitrogen-Doped Carbon Nanomaterials as Non-Metal Electrocatalysts for Water Oxidation. Nat. Commun. 2013, 4, 1-7.

(10) Lai, L.; Potts, J. R.; Zhan, D.; Wang, L.; Poh, C. K.; Tang, C.; Gong, H.; Shen, Z.; Lin, J.; Ruoff, R. S. Exploration of the Active Center Structure of Nitrogen-Doped Graphene-Based Catalysts for Oxygen Reduction Reaction. Energy Environ. Sci. 2012, 5 (7), 79367942.

(11) Guo, D.; Shibuya, R.; Akiba, C.; Saji, S.; Kondo, T.; Nakamura, J. Active Sites of Nitrogen-Doped Carbon Materials for Oxygen Reduction Reaction Clarified Using Model Catalysts. Science (Washington, DC, U. S.) 2016, 351 (6271), 361-365.

(12) Straten, J. W.; Schleker, P.; Krasowska, M.; Veroutis, E.; Granwehr, J.; Auer, A. A.; Hetaba, W.; Becker, S.; Schlögl, R.; Heumann, S. Nitrogen-Functionalized Hydrothermal Carbon Materials by Using Urotropine as the Nitrogen Precursor. Chem. - Eur. J. 2018, 24, 12298-12317.

(13) Zhao, L.; Baccile, N.; Gross, S.; Zhang, Y.; Wei, W.; Sun, Y.; Antonietti, M.; Titirici, M. M. Sustainable Nitrogen-Doped Carbonaceous Materials from Biomass Derivatives. Carbon 2010, 48 (13), 3778-3787.

(14) Titirici, M. M.; White, R. J.; Zhao, L. Nitrogen-Doped Hydrothermal Carbons. Green 2012, 2 (1), 25-40.

(15) Xie, Z.; Huang, B.-B.; Liu, Y. Biomass Derived 2D Carbons via Hydrothermal Carbonization Method as Efficient Bifunctional ORR/ HER Electrocatalysts. J. Mater. Chem. A 2017, 5 (45), 23481-23488.

(16) Ribeiro, R. S.; Silva, A. M. T.; Figueiredo, J. L.; Faria, J. L.; Gomes, H. T. The Influence of Structure and Surface Chemistry of Carbon Materials on the Decomposition of Hydrogen Peroxide. Carbon 2013, 62, 97-108.

(17) Düngen, P.; Schlögl, R.; Heumann, S. Non-Linear Thermogravimetric Mass Spectrometry of Carbon Materials Providing Direct Speciation Separation of Oxygen Functional Groups. Carbon 2018, 130, 614-622.

(18) Kim, U. J.; Furtado, C. A.; Liu, X.; Chen, G.; Eklund, P. C. Raman and IR Spectroscopy of Chemically Processed Single-Walled Carbon Nanotubes. J. Am. Chem. Soc. 2005, 127 (44), 15437-15445.

(19) Apicella, B.; Barbella, R.; Ciajolo, A.; Tregrossi, A. Comparative Analysis of the Structure of Carbon Materials Relevant in Combustion. Chemosphere 2003, 51 (10), 1063-1069.

(20) Baccile, N.; Laurent, G.; Coelho, C.; Babonneau, F.; Zhao, L.; Titirici, M. M. Structural Insights on Nitrogen-Containing Hydrothermal Carbon Using Solid-State Magic Angle Spinning 13C and 15N Nuclear Magnetic Resonance. J. Phys. Chem. C 2011, 115 (18), 8976-8982.

(21) Mao, J.; Cao, X.; Olk, D. C.; Chu, W.; Schmidt-Rohr, K. Advanced Solid-State NMR Spectroscopy of Natural Organic Matter. Prog. Nucl. Magn. Reson. Spectrosc. 2017, 100, 17-51.

(22) Titirici, M.-M.; White, R. J.; Brun, N.; Budarin, V. L.; Su, D. S.; Del Monte, F.; Clark, J. H.; MacLachlan, M. J. Sustainable Carbon Materials. Chem. Soc. Rev. 2015, 44, 250-290.

(23) Botto, R. E.; Wilson, R.; Winans, R. E. Evaluation of the Reliability of Solid 13C NMR Spectroscopy for the Quantitative
Analysis of Coals: Study of Whole Coals and Maceral Concentrates. Energy Fuels 1987, 1 (2), 173-181.

(24) Mao, J.; Chen, N.; Cao, X. Characterization of Humic Substances by Advanced Solid State NMR Spectroscopy: Demonstration of a Systematic Approach. Org. Geochem. 2011, 42 (8), 891902.

(25) Schmidt-Rohr, K.; Spiess, H. Multidimensional Solid-State NMR and Polymers; Academic Press: London, 1994.

(26) Kimmich, R. Tomography, Diffusometry, Relaxometry; SpringerVerlag: Berlin, 1997.

(27) Conte, P.; Marsala, V.; De Pasquale, C.; Bubici, S.; Valagussa, M.; Pozzi, A.; Alonzo, G. Nature of Water-Biochar Interface Interactions. GCB Bioenergy 2013, 5, 116-121.

(28) Conte, P.; Hanke, U. M.; Marsala, V.; Cimò, G.; Alonzo, G.; Glaser, B. Mechanisms of Water Interaction with Pore Systems of Hydrochar and Pyrochar from Poplar Forestry Waste. J. Agric. Food Chem. 2014, 62 (21), 4917-4923.

(29) Brunauer, S.; Emmett, P. H.; Teller, E. Adsorption of Gases in Multimolecular Layers. J. Am. Chem. Soc. 1938, 60 (2), 309-319.

(30) Mao, J.-D.; Schmidt-Rohr, K. Accurate Quantification of Aromaticity and Nonprotonated Aromatic Carbon Fraction in Natural Organic Matter by $13 \mathrm{C}$ Solid-State Nuclear Magnetic Resonance. Environ. Sci. Technol. 2004, 38 (9), 2680-2684.

(31) Fung, B. M.; Khitrin, A. K.; Ermolaev, K. An Improved Broadband Decoupling Sequence for Liquid Crystals and Solids. J. Magn. Reson. 2000, 142 (1), 97-101.

(32) Wang, J. H. Effect of Ions on the Self-Diffusion and Structure of Water in Aqueous Electrolytic Solutions. J. Phys. Chem. 1954, 58 (9), 686-692.

(33) Stejskal, E. O.; Tanner, J. E. Spin Diffusion Measurements: Spin Echoes in the Presence of a Time-Dependent Field Gradient. J. Chem. Phys. 1965, 42 (1), 288-292.

(34) Nilsson, M.; Connell, M. A.; Davis, A. L.; Morris, G. A. Biexponential Fitting of Diffusion-Ordered NMR Data: Practicalities and Limitations. Anal. Chem. 2006, 78 (9), 3040-3045.

(35) Kingsley, P. B. Methods of Measuring Spin-Lattice (T1) Relaxation Times: An Annotated Bibliography. Concepts Magn. Reson. 1999, 11 (4), 243-276.

(36) Brun, N.; García-González, C. A.; Smirnova, I.; Titirici, M. M. Hydrothermal Synthesis of Highly Porous Carbon Monoliths from Carbohydrates and Phloroglucinol. RSC Adv. 2013, 3 (38), 1708817096.

(37) Van Putten, R. J.; Van Der Waal, J. C.; De Jong, E.; Rasrendra, C. B.; Heeres, H. J.; De Vries, J. G. Hydroxymethylfurfural, a Versatile Platform Chemical Made from Renewable Resources. Chem. Rev. 2013, 113 (3), 1499-1597.

(38) Titirici, M.-M.; Antonietti, M.; Baccile, N. Hydrothermal Carbon from Biomass: A Comparison of the Local Structure from Poly- to Monosaccharides and Pentoses/Hexoses. Green Chem. 2008, 10 (11), 1204.

(39) Patil, S. K. R.; Lund, C. R. F. Formation and Growth of Humins via Aldol Addition and Condensation during Acid-Catalyzed Conversion of 5-Hydroxymethylfurfural. Energy Fuels 2011, 25 (10), $4745-4755$.

(40) Demir-Cakan, R.; Baccile, N.; Antonietti, M.; Titirici, M. Carboxylate-Rich Carbonaceous Materials via One-Step Hydrothermal Carbonization of Glucose in the Presence of Acrylic Acid. Chem. Mater. 2009, 21 (3), 484-490.

(41) Falco, C.; Baccile, N.; Titirici, M.-M. Morphological and Structural Differences between Glucose, Cellulose and Lignocellulosic Biomass Derived Hydrothermal Carbons. Green Chem. 2011, 13 (11), 3273.

(42) Harris, R. K.. Nuclear Magnetic Resonance Spectroscopy; Longman Publishing Group: 1986.

(43) Ernst, R. R.; Bodenhausen, A. W.; Wokaun, A. Principles of Nuclear Magnetic Resonance in One and Two Dimensions; Clarendon Press: Oxford, 1987.

(44) Kyakuno, H.; Matsuda, K.; Nakai, Y.; Ichimura, R.; Saito, T.; Miyata, Y.; Hata, K.; Maniwa, Y. Rotational Dynamics and Dynamical 
Transition of Water inside Hydrophobic Pores of Carbon Nanotubes. Sci. Rep. 2017, 7 (1), 14834.

(45) Kleinberg, R. L.; Kenyon, W. E.; Mitra, P. P. Mechanism of NMR Relaxation of Fluids in Rock. J. Magn. Reson., Ser. A 1994, 108, 206-214.

(46) Brownstein, K. R.; Tarr, C. E. Spin-Lattice Relaxation in a System Governed by Diffusion. J. Magn. Reson. 1977, 26 (1), 17-24.

(47) Bloembergen, N.; Purcell, E. M.; Pound, R. V. Relaxation Effects in Nuclear Magnetic Resonance Absorption. Phys. Rev. 1948, 73 (7), 679-712.

(48) Conte, P.; Alonzo, G. Environmental NMR: Fast-Field-Cycling Relaxometry. eMagRes. 1996, 2 (3), 389-398.

(49) Topgaard, D.; Söderman, O. Self-Diffusion of Nonfreezing Water in Porous Carbohydrate Polymer Systems Studied with Nuclear Magnetic Resonance. Biophys. J. 2002, 83 (6), 3596-3606.

(50) Sen, P. N. Time-Dependent Diffusion Coefficient as a Probe of Geometry. Concepts Magn. Reson. 2004, 23A (1), 1-21.

(51) Karunanithy, G.; Wheeler, R. J.; Tear, L. R.; Farrer, N. J.; Faulkner, S.; Baldwin, A. J. An In-Cell Diffusion Method to Characterize the Size, Abundance and Permeability of Cells. J. Magn. Reson. 2019, 302, 1.

(52) Caldarelli, S. Chromatographic NMR: A Tool for the Analysis of Mixtures of Small Molecules. Magn. Reson. Chem. 2007, 45, S48S55.

(53) Laage, D.; Elsaesser, T.; Hynes, J. T. Water Dynamics in the Hydration Shells of Biomolecules. Chem. Rev. 2017, 117 (16), 10694-10725.

(54) Sahu, S.; Zwolak, M. Access Resistance in Graphene Nanopores. Phys. Chem. Chem. Phys. 2018, 20, 4646. 